\title{
Proactive management of histopathology workloads: analysis of the UK Royal College of Pathologists' recommendations on specimens of limited or no clinical value on the workload of a teaching hospital gastrointestinal pathology service
}

\section{S S Cross, J L Stone}

See end of article for

J Clin Pathol 2002;55:850-852

authors' affiliations

Correspondence to: Dr S S Cross, Academic Unit of Pathology, Section of Oncology and Pathology, Division of Genomic Medicine, School of Medicine and

Biomedical Science, University of Sheffield Beech Hill Road, South Yorkshire S10 2RX, UK ; s.s.cross@sheffield.ac.uk

Accepted for publication 12 June 2002

\begin{abstract}
Aims: To investigate the effect on the workload of a gastrointestinal pathology service of implementing the recommendations of the Royal College of Pathologists' (RCPath) working party on specimens of limited or no clinical value (LONCV).

Methods: All endoscopic gastrointestinal pathology reports for the first three months of 2001 at a large teaching hospital were reviewed against the RCPath recommendations. Specimens in the category of LONCV were recorded and the final histopathology diagnosis noted.

Results: The biopsies in the LONCV category were $30 \%$ of oesophageal, $61 \%$ of gastric, $0.5 \%$ of duodenal, and $7 \%$ of colorectal origin.

Conclusions: Implementing the RCPath recommendations would reduce the number of requests for the examination of gastrointestinal endoscopic specimens by 3500 specimens each year in this department. None of the specimens in the LONCV category showed an abnormality that could not have been detected by a more efficient and less invasive method. In the UK, where there is a severe shortage of trained histopathologists, the implementation of these recommendations would ensure that these scarce resources are not misused.
\end{abstract}

$\mathrm{H}$ istopathology, like other laboratory medicine specialties, ${ }^{12}$ has been a demand led specialty responding to the work sent for examination. ${ }^{3}$ This has led to many imbalances between demand and histopathology resources, with health care managers often making no extra provision for histopathology, despite appointing additional staff who generate histopathology work (for example, consultant gastroenterologists, endoscopy nurses, etc). At the same time, there has been an intrinsic increase in histopathology work because of the increased information that is included in reports, ${ }^{4}$ often stimulated by minimum dataset guidelines from national pathology organisations. ${ }^{5}$ In the UK, there has been an additional problem because there has been an imbalance between the number of trainees and the number of consultant post vacancies, so that at present $20 \%$ of consultant histopathologist posts in the UK are unfilled.

All these factors suggest that histopathologists should take a proactive role in the management of their workload. As part of its recovery plan for histopathology, ${ }^{6}$ the Royal College of Pathologists (RCPath) in the UK has recently convened a working group to make an evidence based evaluation of investigations of doubtful usefulness, which make little or no contribution to patient care and welfare. This group has produced a report of its findings. ${ }^{7}$ In this report there are many categories of gastrointestinal specimens that have been found to be of little or no clinical value, including oesophageal and gastric biopsies from endoscopically normal organs and gastric biopsies for the sole purpose of detecting Helicobacter pylori. Because gastrointestinal specimens constitute a large proportion of the workload in most histopathology departments, the application of these guidelines could result in significant workload reductions. This study makes a retrospective evaluation of the effect of applying these guidelines to the

gastrointestinal pathology specimens received in a large department of histopathology.

\begin{abstract}
"There has been an additional problem because there has been an imbalance between the number of trainees and the number of consultant post vacancies, so that at present $20 \%$ of consultant histopathologist posts in the UK are unfilled"
\end{abstract}

\section{MATERIALS AND METHODS}

All endoscopic gastrointestinal biopsies received in the first three months of 2001 by the department of histopathology at the Royal Hallamshire Hospital, Sheffield were reviewed. Biopsies referred from other centres for specialist review were excluded. Cases were divided according to anatomical site- that is, oesophageal, gastric, duodenal, or colorectal. For uniformity, the number of requests was recorded, rather than the number of patients or number of biopsies. This was because some cases had more than one request for each patient and some requests involved multiple biopsies grouped together in one pot as a single request. Requests were classified as either "valid" or "invalid" according to the working group's guidelines. Any significant abnormalities found in the invalid requests were noted. The number of requests where multiple random colonic biopsies were submitted in separate pots were counted. A separate tally was made of the number of requests that this figure could potentially be reduced to if following the working group's guidelines for such

Abbreviations: LONCV, specimens of limited or no clinical value; RCPath, Royal College of Pathologists 
Table 1 Numbers of "valid" and "invalid" requests at each organ site

\begin{tabular}{|c|c|c|c|c|c|c|c|c|}
\hline & \multicolumn{2}{|c|}{ Oesophageal } & \multicolumn{2}{|c|}{ Gastric } & \multicolumn{2}{|c|}{ Duodenal } & \multicolumn{2}{|c|}{ Colonoscopic } \\
\hline & $\mathrm{N}$ & $\%$ & $\mathrm{~N}$ & $\%$ & $N$ & $\%$ & $\mathrm{~N}$ & $\%$ \\
\hline Total requests & 184 & & 286 & & 378 & & 966 & \\
\hline Valid requests & 127 & 69.0 & 108 & 37.8 & 364 & 96.3 & 891 & 92.2 \\
\hline Invalid requests & 56 & 30.4 & 175 & 61.2 & 2 & 0.5 & 64 & 6.6 \\
\hline No clinical details given & 1 & 0.6 & 3 & 1.0 & 13 & 3.2 & 11 & 1.1 \\
\hline Clinically relevant abnormalities in invalid requests & 3 & & 0 & & 0 & & 1 & \\
\hline
\end{tabular}

\begin{tabular}{|lrr|}
\hline Table 2 Categories of "invalid" requests in each organ site & \\
\hline Organ & Reason for invalidity & $\mathrm{N}$ \\
\hline Oesophagus & Biopsy of normal oesophagus & 3 \\
& Biopsy for reflux oesophagitis without discrete lesion & 50 \\
& Biopsy for so called ultra short Barrett's oesophagus & 3 \\
& Total & 56 \\
Stomach & Biopsy of normal stomach & 16 \\
& Biopsy for Helicobacter pylori & 68 \\
& Biopsy for gastritis/intestinal metaplasia without a discrete lesion & 91 \\
& Total & 175 \\
& Biopsy not related to the investigation of coeliac disease & 2 \\
Duodenum & Total & 2 \\
& & \\
& Terminal ileum biopsy & 26 \\
Colorectum & Normal scope, no diarrhoea & 26 \\
& Random rectal biopsy for rectal bleeding & 12 \\
& Total & 64 \\
\hline
\end{tabular}

biopsies to be submitted in two separate pots (left side and right side of the colon).

The guidelines gave no specific age limit for "the correct clinical setting" for biopsies of normal appearing colon in the investigation of persistent watery diarrhoea. Therefore, all requests matching these criteria (regardless of age) were deemed valid for the purposes of this review. Incidentally, most of these cases fell into the suggested range of "middle aged or elderly".

\section{RESULTS}

The results are summarised in tables 1 and 2 .

\section{Oesophageal biopsies}

A review of these biopsies revealed a high proportion of invalid requests $(30.4 \%)$, particularly of biopsies for reflux oesophagitis without a discrete lesion. Three clinically relevant abnormalities were reported in the 56 invalid requests. All three showed columnar lined lower oesophagus.

\section{Gastric biopsies}

A large proportion of these biopsies were classified as invalid $(61.2 \%)$. Biopsies for Helicobacter pylori and biopsies of gastritis without a focal abnormality were the most frequent categories. No further clinically relevant abnormalities were found in the invalid requests.

\section{Duodenum}

Most of these requests were valid. Exclusion of, diagnosis of, and the follow up of coeliac disease were the main indications for duodenal biopsy.

\section{Colonoscopic biopsies}

A small proportion of these requests were invalid (6.6\%). This proportion would have been slightly higher if a strictly defined cut off age for random colon biopsies for microscopic colitis was included in the review criteria. One clinically relevant abnormality was found in an invalid request. This was a finding of ulcerative colitis in random rectal biopsies for rectal bleeding. In total, 387 valid random colonic biopsies from different sites were submitted in the three months. If these were submitted collectively in right and left pots, as advised by the guidelines, this figure would be reduced to 184 . This is a reduction in workload by 203 requests over three months.

\section{DISCUSSION}

These results show that a considerable proportion of the endoscopic gastrointestinal biopsies submitted for examination in a large teaching hospital fall into the category of specimens with limited or no clinical value (LONCV) as defined in the RCPath report. ${ }^{7}$ They also show that very few clinically relevant abnormalities were found in the specimens that fell into that category. Three oesophageal specimens were reported as showing columnar lined lower oesophagus, but this diagnosis is dependent on biopsies being taken from above the anatomical gastro-oesophageal junction. We cannot exclude the possibility that these biopsies were taken from below the gastro-oesophageal junction or that they represented so called ultrashort segment Barrett's oesophagus, for which the RCPath report does not recommend histopathology examination. There was one case of ulcerative colitis diagnosed in a patient where the clinical details on the request form only recorded rectal bleeding. It is likely that the endoscopic appearances in this case were abnormal and it would therefore represent a valid biopsy.

"It is a significant reduction and if this was extended across the UK, and to other categories of histopathology specimens, it would represent a significant reduction in workload, which would ease the current staffing crisis" 


\section{Take home messages}

- By extrapolation of results, we calculate that implementing the recommendations of the Royal College of Pathologists would reduce the number of requests for the examination of gastrointestinal endoscopic specimens by 3500 specimens each year in our department

- None of the specimens in the specimens of limited or no clinical value category showed an abnormality that could not have been detected by a more efficient and less invasive method

- In the UK, where there is a severe shortage of trained histopathologists, the implementation of these recommendations would ensure that these scarce resources are not misused

- To achieve these reductions, a multidisciplinary dialogue needs to be opened between clinicians who use histopathology services, healthcare managers, and histopathologists and conflicting guidelines will need to be resolved

If all the invalid specimens had not been reported and the individual colonic biopsies combined into right and left sided pots then there would have been a reduction in specimen requests of 862 in the first three months of 2001. This extrapolates to a reduction of 3500 requests each year. It is difficult to express this in terms of whole time consultant histopathologists in a UK teaching hospital because the RCPath recommended workloads are for an overall mixed workload. The specimens in our study that would be eliminated by applying the recommendations of the Howat report are all of low complexity, so it would be incorrect to calculate that 3500 specimens equates to the workload of $13 / 4$ whole time consultant histopathologists in a UK teaching hospital. However, it is still a significant reduction and if this was extended across the UK, and to other categories of histopathology specimens, ${ }^{7-10}$ it would represent a significant reduction in workload, which would ease the current staffing crisis. There is a similar staffing crisis among biomedical scientists working in histopathology laboratories in the UK and the elimination of specimens of LONCV would also provide some alleviation of this.

This is a retrospective analysis which shows that significant reductions in histopathology workload can be achieved by applying the recommendations of the RCPath's working party on histopathology of limited or no clinical value. To achieve these reductions, a multidisciplinary dialogue needs to be opened between clinicians who use histopathology services, healthcare managers, and histopathologists. There will have to be some resolution of conflicting guidelines-for example, some gastroenterology guidelines recommend biopsy of the terminal ileum to "prove" complete colonoscopic examination, which conflicts with the RCPath's recommendations. ${ }^{11-13}$ Nevertheless, there is considerable scope for a more proactive role for histopathologists in workload management.

\section{Authors' affiliations}

S S Cross, Academic Unit of Pathology, Section of Oncology and Pathology, Division of Genomic Medicine, School of Medicine and Biomedical Science, University of Sheffield, South Yorkshire S10 2RX, UK J L Stone, Department of Histopathology, Royal Hallamshire Hospital, Sheffield Teaching Hospitals NHS Trust, Glossop Road, Sheffield S10 2UL, UK

\section{REFERENCES}

1 Cartwright $\mathrm{K}$, Lewis D, Roberts $\mathrm{C}$, et al. Workload and stress in consultant medical microbiologists and virologists: a questionnaire survey. J Clin Pathol 2002;55:200-5

2 Shirley J, Wing S. Workload, organisation, and cost of haematology laboratory out of hours services. J Clin Pathol 2001;54:647-9.

3 Griffiths DF. Workload measurement in histopathology. J Clin Pathol 1999;52:398.

4 Cross SS, Bull AD. Is the informational content of histopathological reports increasing? J Clin Pathol 1992;45:179-80.

5 Royal College of Pathologists. Standards and minimum datasets for reporting cancers. http://www.rcpath.org/activities/minimum_list.html accessed on 8 April 2002.

6 Underwood JCE. A recovery plan for histopathology. RCPath Bulletin $2001 ; 113: 12-14$

7 Howat A, Boyd K, Douce G, et al. SAC on histopathology working group on histopathology of limited or no clinical value. Royal College of Pathologists 2002. http://www.rcpath.org/fellows_members/ howatdoc_171201.doc accessed on 8 April 2002.

8 Burton JL, Goepel JR, Lee JA. Demand management in urine cytology: a single cytospin slide is sufficient. J Clin Pathol 2000;53:718-19.

9 Burton JL, Lee JA, Goepel JR. Successful demand management in histopathology: a model using sputum cytology. J Clin Pathol 2001;54:896.

10 Chakrabarty A, Franks AJ. Temporal artery biopsy: is there any value in examining biopsies at multiple levels? J Clin Pathol 2000;53:131-6.

11 Bini EJ. Endoscopic approach to HIV-associated diarrhea: how far is far enough? Am J Gastroenterol 1999;94:556-9.

12 Greff $\mathbf{M}$. Enteroscopy-endoscopic guidelines. Endoscopy 1998;30:641-2.

13 British Society for Gastroenterology. Training programmes in endoscopy. http://www.bsg.org.uk/trainee/train_prog.htm accessed on 8 April 2002 